\title{
Development of fast, background-limited transition-edge sensors for the Background-Limited Infrared/Sub-mm Spectrograph (BLISS) for SPICA
}

\author{
Andrew D. Beyer ${ }^{a^{*}}$, M. Kenyon ${ }^{a}$, P.M. Echternach ${ }^{\mathrm{b}}$, B. Bumble ${ }^{\mathrm{b}}$, M. C. Runyan ${ }^{\mathrm{b}}$,T. Chui ${ }^{\mathrm{b}}$, C.M. \\ Bradford $^{\mathrm{a}, \mathrm{b}}$, W.A. Holmes ${ }^{\mathrm{a}}$, and J.J. Bock ${ }^{\mathrm{a}, \mathrm{b}}$. \\ ${ }^{a} J e t$ Propulsion Laboratory, California Institute of Technology 4800 Oak Grove Dr., Pasadena, CA \\ USA 91109; ${ }^{\mathrm{b}}$ California Institute of Technology, 1200 E. California Blvd. Pasadena, CA USA \\ 91125
}

\begin{abstract}
We report experimental progress toward demonstrating background-limited arrays of membrane-isolated transition-edge sensors (TESs) for the Background Limited Infrared/Sub-mm Spectrograph (BLISS). BLISS is a space-borne instrument with grating spectrometers for wavelengths $\lambda=35-435 \mu \mathrm{m}$ and with $\mathrm{R}=\lambda / \Delta \lambda \sim 500$. The goals for BLISS TESs are: noise equivalent power (NEP) $=5 \times 10^{-20} \mathrm{~W} / \mathrm{Hz}^{1 / 2}$ and response time $\tau<30 \mathrm{~ms}$. We expect background-limited performance from bilayers TESs with $\mathrm{T}_{\mathrm{C}}=65 \mathrm{mK}$ and $\mathrm{G}=15 \mathrm{fW} / \mathrm{K}$. However, such TESs cannot be operated at $50 \mathrm{mK}$ unless stray power on the devices, or dark power $P_{D}$, is less than $200 \mathrm{aW}$. We describe criteria for measuring $P_{D}$ that requires accurate knowledge of $T_{C}$. Ultimately, we fabricated superconducting thermistors from $\operatorname{Ir}\left(T_{C} \geq 135 \mathrm{mK}\right)$ and $\mathrm{Mo} / \mathrm{Cu}$ proximitized bilayers, where $\mathrm{T}_{\mathrm{C}}$ is the thermistor transition temperature. We measured the Ir TES arrays in our $45 \mathrm{mK}$ base temperature adiabatic demagnetization refrigerator test system, which can measure up to eight $1 \times 32$ arrays simultaneously using a time-division multiplexer, as well as our single-pixel test system which can measure down to $15 \mathrm{mK}$. In our previous Ir array measurements our best reported performance was $\mathrm{NEP}=2.5 \times 10^{-19} \mathrm{~W} / \mathrm{Hz}^{1 / 2}$ and $\tau \sim 5 \mathrm{~ms}$ for straight-beam TESs. In fact, we expected NEP $\sim 1.5 \times 10^{-19} \mathrm{~W} / \mathrm{Hz}^{1 / 2}$ for meander beam TESs, but did not achieve this previously due to $1 / \mathrm{f}$ noise. Here, we detail improvements toward measuring the expected NEP and demonstrate $\mathrm{NEP}=(1.3 \pm 0.2) \times 10^{-19} \mathrm{~W} / \mathrm{Hz}^{1 / 2}$ in our single-pixel test system and $\mathrm{NEP}=(1.6 \pm 0.3) \times 10^{-19} \mathrm{~W} / \mathrm{Hz}^{1 / 2}$ in our array test system.
\end{abstract}

Keywords: transition-edge sensors, infrared spectrometer, sub-mm spectrometer, photon background-limited

\section{INTRODUCTION}

The Background-Limited Infrared/Sub-mm Spectrograph (BLISS) is a proposed instrument to fly on the JAXA satellite mission known as SPICA. BLISS is a broadbroad grating spectrometer divided into six bands spanning $35 \mu \mathrm{m}$ to $435 \mu \mathrm{m}$. In order to be photon background-limited and to optically chop the signal from 1 to $5 \mathrm{~Hz}$ for noise reduction, detectors on BLISS need to at least demonstrate noise equivalent power (NEP) equal to $10^{-19} \mathrm{~W} / \mathrm{Hz}$ and response time $\tau$ of $\sim 150 \mathrm{~ms}$. The goal for BLISS is to have NEP $=5 \times 10^{-20} \mathrm{~W} / \mathrm{Hz}^{1 / 2}$ and $\tau<30 \mathrm{~ms}$. Membrane-isolated transition-edge sensors (TESs) are a natural candidate to meet these requirements, and we report our progress toward demonstrating these specifications in our TESs.

\subsection{Expected photon noise for BLISS on SPICA}

Observing from a space-based platform gives BLISS a significant advantage over ground based IR/sub-mm observatories, as the photon background noise is greatly reduced in space and the atmosphere does not absorb and prevent IR/sub-mm light from reaching the spectrometer. The expected photon power due to the SPICA telescope, the telescope baffles, the CMB, and the interstellar and interplanetary dust ranges from $0.1 \mathrm{aW}$ to $3 \mathrm{aW}$ over the six bands of BLISS. ${ }^{1} \quad$ Including a safety factor of 75 to 150 , the worst-case photon power expected ranges from about $50 \mathrm{aW}$ to 200aW. BLISS is designed and specified so that the detector NEP is equal to the expected photon noise at the instrument without safety factor, while simultaneously having sufficient dynamic range to accommodate the safety factor. (In reality, a dual-TES approach using a Ti TES in series with the main sensing TES will be employed to guarantee dynamic range from 3000 to 15000 , depending on the band.) Photon noise is generally described by shot and

*andrew.d.beyer@jpl.nasa.gov; phone 1626-434-6868;

Millimeter, Submillimeter, and Far-Infrared Detectors and Instrumentation for Astronomy VI, edited by Wayne S. Holland, Jonas Zmuidzinas, Proc. of SPIE Vol. 8452

84520G ( 2012 SPIE · CCC code: 0277-786X/12/\$18 - doi: 10.1117/12.926326 
Bose noise according to

$$
p_{n}^{2}=2 h\langle v\rangle P_{o p t}+\frac{P_{o p t}^{2}}{2\langle\Delta v\rangle},
$$

where $\mathrm{p}_{\mathrm{n}}{ }^{2}$ is the expected photon NEP squared, $\mathrm{P}_{\mathrm{opt}}$ is the photon power, $<v>$ is the average frequency of the light, and $\left\langle\Delta v>\right.$ is the average bandwidth of the light. The term linearly proportional to $\mathrm{P}_{\mathrm{opt}}$ is the shot-noise term, while the term proportional to $\mathrm{P}_{\mathrm{opt}}{ }^{2}$ is the Bose term. The Bose term is subdominant to the shot noise for the in-band light in the space environment for all frequencies above the microwave background peak. The expected photon noise ranges from $2 \times 10^{-20}$ $\mathrm{W} / \mathrm{Hz}^{1 / 2}$ (shortest $5 \lambda$ bands) to $5 \times 10^{-20} \mathrm{~W} / \mathrm{Hz}^{1 / 2}$ (longest $\lambda$ band). Membrane-isolated transition-edge sensors (TESs) are a natural candidate to meet the detector NEP requirement (equal to the photon NEP) and to be formatted into arrays that cover the full band at a reasonable resolving power for BLISS, $\mathrm{R}=\lambda / \Delta \lambda \sim 500$, requiring about 4000 TESs readout with a time-domain multiplexer.

The spectroscopic line sensitivity of far-IR/sub-mm spectrometers determines the ultimate performance and ability to meet the BLISS scientific objectives. The matching of the TES detector $\mathrm{NEP}_{\text {det }}$ to the photon background $\mathrm{NEP}_{\text {opt }}$ given an instrument with $\mathrm{R} \sim 500$, leads to the best possible performance. BLISS will couple a single source to the six individual bands which combine to cover the 38-430 micron range and will chop the signal between two spectrometers. SPICA can be expected to provide $75 \backslash \%$ aperture efficiency on a 3.15-meter telescope and with BLISS we will expect $25 \%$ end-to-end transmission through to the detectors. This estimated value is based on the performance of spectrometers such as Z-spec and IRS on Spitzer, which are similar to the BLISS spectrometer modules. ${ }^{2,3}$ The $5 \sigma$ 2hour line sensitivity is $10^{-20} \mathrm{~W} / \mathrm{m}^{2}$ for all 6 bands, assuming a detector NEP of $5 \times 10^{-20} \mathrm{~W} / \mathrm{Hz}^{1 / 2}$.

\subsection{Experimental design criteria for TES testing}

The expected NEP for a TES bolometer is given by

$$
N E P=\sqrt{4 k_{B} T_{C}^{2} G \gamma} \text { and } \gamma=\frac{n+1}{2 n+3} \frac{\left(1-(1-t)^{2 n+3}\right)}{\left(1-(1-t)^{n+1}\right)},
$$

where $k_{B}$ is Boltzmann's constant, $G$ is the thermal conductance of the TES support beams, and $T_{C}$ is the transition temperature of the superconducting thermistor. $G$ varies with $T$ according to $G \sim \mathrm{T}^{\mathrm{n}}$ and $\gamma$ accounts for thermal gradients along the support beams. Here, $t$ is $1-T_{o p} / T_{C}$, where $T_{o p}$ is the operating temperature of the TES. Given a space-qualified cryocooler operating at $50 \mathrm{mK}$, a $\mathrm{T}_{\mathrm{C}}=65 \mathrm{mK}$, and $\mathrm{G} \sim \mathrm{T}^{1 / 2}$ for BLISS TESs ${ }^{1}$, we expect to meet the NEP goal at $15 \mathrm{fW} / \mathrm{K}$.

The heat flow from the TES membrane to the substrate, while operating the TES within the transition at $\mathrm{T}_{\mathrm{C}}$, may be modeled by:

$$
P_{\text {flow }}=K\left(T_{C}^{n+1}-T_{o p}^{n+1}\right), G=d P_{\text {flow }} / d T,
$$

where $\mathrm{K}$ is a constant that may be related to G. A background-limited TES at margin will have a heat outflow of 200aW at $50 \mathrm{mK}$. Thus, the stray power, or dark power $\mathrm{P}_{\mathrm{D}}$, needs to be $<200 \mathrm{aW}$ to operate at $50 \mathrm{mK}$, because this $\mathrm{P}_{\mathrm{D}}$ is the maximum heat outflow that can flow out of the device support beams without driving the TES from within the transition to the normal state. Optical and electrical sources contribute to $\mathrm{P}_{\mathrm{D}}$, which include stray optical radiation, SQUID amplifier Josephson oscillations acting back on the TES, and stray electrical power coming down the bias lines.

\section{EXPERIMENTAL SETUP}

\subsection{BLISS dark testing: single-pixel and array test setups}

We employ two testing systems to characterize TESs for BLISS: a single-pixel dilution refrigerator setup and a multiplexed array test system utilizing a dual-stage adiabatic demagnetization refrigerator (ADR) with $1 \mathrm{~K}$ and $40 \mathrm{mK}$ stages. The single-pixel test system has a base temperature of $15 \mathrm{mK}$, while the ADR array test system has a base temperature of $45 \mathrm{mK}$ at the array test focal plane. Both test systems utilize the typical voltage-biased feedback setup for operating a TES within the transition at $\mathrm{T}_{\mathrm{C}}$ using negative electrothermal feedback (ETF). ${ }^{4} \mathrm{~A}$ single SQUID amplifier 
reads out the current through the TES in the single-pixel test system, and $1.5 \mathrm{~m} \Omega$ shunt resistors are used to bias the TES. A SQUID time-domain multiplexer (TDM) is utilized in the array test system. The multiplexed SQUID chips and shunt chips needed to operate the TDM were provided by NIST. We installed two varieties of MUX chips, denoted as MUX05 and MUX09 chips ${ }^{5}$, as well as two types of shunt chips with $160 \mu \Omega$ and $3 \mathrm{~m} \Omega$ impedances. A general rule is that the shunt resistance should be less than or equal to $1 / 10$ of the normal state resistance $R_{N}$ of the TES superconducting thermistor. Our Ir TESs had $\mathrm{R}_{\mathrm{N}} \sim 55 \mathrm{~m} \Omega$, while our Mo/Cu TES had $\mathrm{R}_{\mathrm{N}}$ of $7 \mathrm{~m} \Omega$ to $14 \mathrm{~m} \Omega$, thus different shunt chips were necessary. We denote the shunt chips as NYQ chips, as the inductances are typically chosen on these chips to reduce aliasing penalties using analysis regarding the Nyquist sampling frequency from L/R time constants.

Both test systems have means to mitigate stray optical/electrical power from affecting the TES operation. As has been reported previously, we employ attenuating coaxial bias lines, a light-tight $\mathrm{Nb}$ can, and RF filters at $1.9 \mathrm{MHz}$ and $200 \mathrm{MHz}$ in our single-pixel test system, and we can achieve $\mathrm{P}_{\mathrm{D}}<250 \mathrm{aW}$ in this test system. ${ }^{6}$ In our current array test system, we employ a light-tight $\mathrm{Nb}$ box, whose electrical feed-through connections are sealed with In gaskets, to shield the TESs from stray optical light. To combat stray electrical power, we employ electrical filters fabricated on Si for stray electrical noise reduction. We have tested both $\mathrm{L} / \mathrm{R}$ and $\mathrm{L} / \mathrm{C}$ filters in the array system. For both designs, the filters are cascaded three times, and the $\mathrm{L} / \mathrm{C}$ filter rolls-off at $\mathrm{f}=15 \mathrm{MHz}$, while the $\mathrm{L} / \mathrm{R}$ filter rolls-off at $\mathrm{f}=150 \mathrm{kHz}$. The narrower noise bandwidth for the $L / R$ filter led to $P_{D} \sim 1 \mathrm{fW}$, while the $L / C$ filter demonstrated $P_{D} \sim 5 \mathrm{fW}$ from previous measurements. ${ }^{7}$ We have recently tested Eccosorb ${ }^{8}$ on top of the L/R filter to absorb RF optical radiation in order to reduce optical stray light. The Eccosorb was placed on top of the filters and was separated by a $\mathrm{Nb}$ partition from the arrays. However, we observed that many devices did not transition in this setup, which we believe was a result of stray magnetic field from the Eccosorb leaking to the TES arrays despite the partition. We have subsequently removed the Eccosorb. Additionally, we built new L/R filters with expected roll-off around $20 \mathrm{kHz}$ with reduced inductance $(\sim 7 \mu \mathrm{H})$ compared to the previously used L/R filters $(\sim 21 \mu \mathrm{H})$ to increase bandwidth and operating range for diagnostic purposes.

\subsection{Determining $\mathbf{P}_{\mathrm{D}}$}

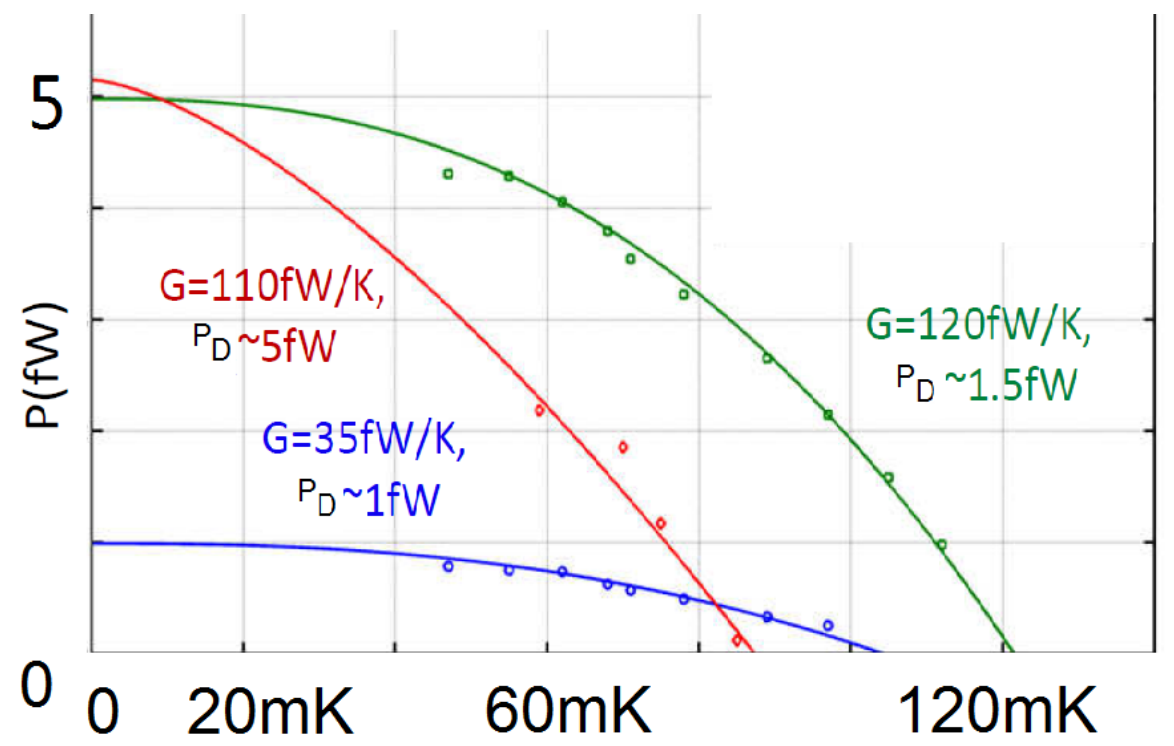

Figure 1: Examples of measured $P$ vs. $T$ curves for $I r$ TESs with different $G$ values and different values of $P_{D}$. We have observed that the $135 \mathrm{mK} \mathrm{T}_{\mathrm{C}}$ of our $\mathrm{XeF}_{2}$ etched devices is not affected by the longitudinal proximity effect down to $10 \mu \mathrm{m}$ wide devices in independent $R$ vs. $T$ measurements. Without knowledge of $T_{C}$, it would incorrectly be assumed that $T_{C}<135 \mathrm{mK}$ for each of the shown devices, as $T_{C}$ is often identified as the temperature at which $P \rightarrow 0$.

In our previous measurements, we have demonstrated the ability to measure $\mathrm{P}_{\mathrm{D}}$ using both Johnson noise Thermometry Devices (JTDs) and TESs in the same experimental setup. A JTD employs two thin-film resistors on a membraneisolated thermal structure. One resistor is used as a thermometer and the other resistor is used as a heater in order to measure $\mathrm{G}$ and the heat capacity $\mathrm{C}$ of the membrane-isolated JTD structure as a function of temperature. Typically, the 
JTD is built out of $\mathrm{Si}_{\mathrm{x}} \mathrm{N}_{\mathrm{y}}(\mathrm{Si}-\mathrm{N})$ support beams and with an $\mathrm{Si}-\mathrm{N}$ absorber similar to the desired TES architecture of BLISS TESs or TESs for similar instruments. This setup allows the thermal structure to be diagnosed over a larger temperature range. A measurement with a JTD in the same setup as a TES is straightforward, as the $\mathrm{P}_{\mathrm{D}}$ incident on a TES may be estimated from the value of $P_{D}$ on the JTD. A JTD measures $P_{D}$ using the measured $G$ as a function of T while measuring the temperature difference between the JTD thermometer versus the base temperature of the test system. More details may be found in Ref.[6].

Our array test system does not employ a JTD, as the many electrical connections designed to connect to the MUX/NYQ chips are not compatible with JTD measurements. Alternatively, the value of $\mathrm{P}_{\mathrm{D}}$ in the array test system may be determined from an accurate determination of $T_{C}$ for the superconducting thermistor on the TES. In our previous measurements on Ir TESs with $T_{C}=135 \mathrm{mK}$, we found $P_{D}$ from $\sim 1 \mathrm{fW}$ to $\sim 5 \mathrm{fW} .^{7}$ The values of $P_{D}$ were determined from the shifting of the outflow power $\mathrm{P}_{\text {flox }}$ (Eq. (3)) curve of a TES according to

$$
P_{\text {observed }}=K\left(T_{C}^{n+1}-T_{o p}^{n+1}\right)-P_{D}
$$

With accurate knowledge of $T_{C}$, determination of $P_{D}$ from Eq. (4) may be found by fitting $P_{\text {observed }}$ as a function of $T$ and by using a value of $\mathrm{n}$ known for device architecture. For our Ir TESs released by $\mathrm{XeF}_{2}$, the value of $\mathrm{T}_{\mathrm{C}}$ is robust over time and size of the thermistor, and $\mathrm{P}_{\mathrm{D}}$ is accurately determined. Without knowledge of $\mathrm{T}_{\mathrm{C}}, \mathrm{T}_{\mathrm{C}}$ may easily be confused with the base temperature at which $P_{D}$ alone is enough to drive the TES into the normal state. An example of different values of $P_{D}$ and their effect on Joule power $P$ as a function of $T$ for Ir devices $\left(T_{C} \sim 135 \mathrm{mK}\right)$ with varying $G$ values is shown in Fig. 1. It should be noted that $P_{D}$ and $T_{C}$ are degenerate variables when fitting in Eq. (4). If one does not know $T_{C}$ or $P_{D}$, then a fit to $P_{\text {observed }}$ for a given value of $n$ may be made using any value of $P_{D}>0$ with a $T_{C}$ such that the constant $\mathrm{KT}_{\mathrm{C}}{ }^{\mathrm{n}+1}-\mathrm{P}_{\mathrm{D}}$ is kept constant. The value of $\mathrm{n}$ may be bounded by differentiating Eq. (4) and by finding what values of $n$ can fit the derivative as a function of $T_{\text {op }}$ within uncertainty. However, knowledge of $T_{C}$ is essential in order to gauge $\mathrm{P}_{\mathrm{D}}$ accurately.

\subsection{Complications in determining $P_{D}$ for bilayer samples}

BLISS will ultimately deploy with bilayer superconducting thermistors, with the bilayer $T_{C}$ tuned via the proximity effect to $T_{C}=65 \mathrm{mK}$. The proximity effect arises when a thin superconducting film and a thin normal metal film are fabricated in contact with each other, and the resulting bilayer $T_{C}$ may be made smaller than $T_{C}$ of the superconducting film alone by tuning the thicknesses of the two layers. Here, thin is generally regarded as a thickness less than the coherence length of the superconducting film.

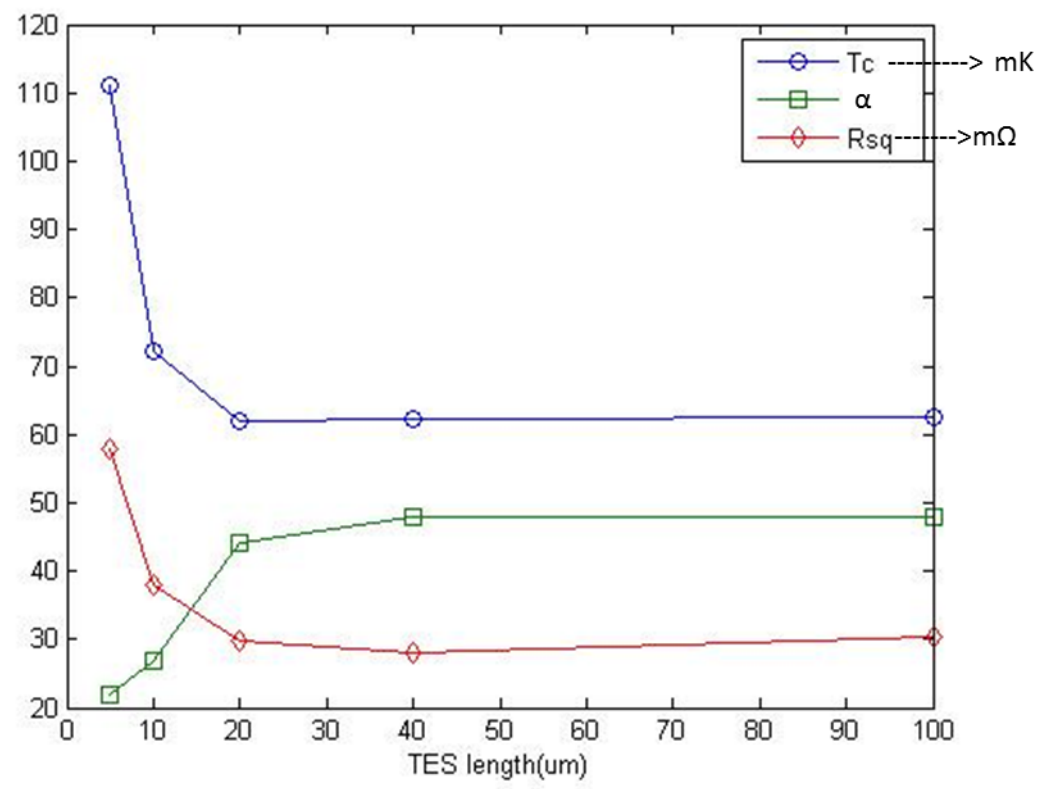

Figure 2: Average values of $T_{C}, \alpha$, and $R_{s q}$ (resistance per square) as a function of TES length for our $M_{0} / C u T_{C} \approx 60 \mathrm{mK}$ recipe. $T_{C}$ is displayed in $m K, \alpha$ is dimensionless, and $R_{s q}$ is in $m \Omega$. 
We have developed a recipe for $\mathrm{Mo} / \mathrm{Cu}$ bilayers with lower $\mathrm{T}_{\mathrm{C}}=60 \mathrm{mK}$; however, longitudinal proximity effects (LPEs) ${ }^{9}$ raise $T_{C}$ above our target $T_{C}$ for shorter TES lengths. Short TES lengths are needed to produce fractional square thermistors with low $\mathrm{R}_{\mathrm{N}}$ for improved responsivity, reduction of multiplexing penalties, and to fit into our Si-N support architecture. Additionally, the sharpness of the superconducting transition at $\mathrm{T}_{\mathrm{C}}$, denoted by $\alpha=\mathrm{d} \operatorname{logR} / \mathrm{d} \log \mathrm{T}$, is also degraded for shorter thermistor lengths. The sharpness is important in achieving the speed goals of BLISS, as the electrothermal feedback speeds up the device below the natural thermal time constant $\tau_{0}=\mathrm{C} / \mathrm{G}$ to $\tau=\tau_{0} /\left(1+\mathrm{P}_{\mathrm{J}} \alpha / \mathrm{GT}\right)$, where $\mathrm{P}_{\mathrm{J}}$ is the Joule power on transition. Previously reported analysis using the Usadel equation ${ }^{10}$ provides a framework for modeling the $\mathrm{T}_{\mathrm{C}}$ for a bilayer and a general physical understanding.

The actual value of $\mathrm{T}_{\mathrm{C}}, \mathrm{R}_{\mathrm{N}}$ and $\alpha$ for a given thermistor are sensitive to geometry due to the LPE. The LPE can be thought of as an unintended proximity effect between the TES thermistor and the superconducting wiring used in the electrothermal circuit to readout the TES. The edges of the thermistor become superconducting due to the proximity of the wiring, which has a higher $\mathrm{T}_{\mathrm{C}}$ than that of the bilayer, raising the temperature at which the thermistor shorts out. We have developed a recipe for $\mathrm{T}_{\mathrm{C}}=60 \mathrm{mK}$ using $\mathrm{Mo} / \mathrm{Cu}$ bilayers.. However, we have discovered that $\mathrm{T}_{\mathrm{C}}$ increases from $60 \mathrm{mK}$ up to $110 \mathrm{mK}$ as the width of the thermistor is decreased below $20 \mu \mathrm{m}$ down to $5 \mu \mathrm{m}$, as shown in Fig. 2. The variation of $\alpha$ and $R_{\mathrm{sq}}$, the resistance per square, are also shown as a function of thermistor length in Fig. 2.

\subsection{Determining $\mathbf{P}_{\mathrm{D}}$ for BLISS TESs}

Given the complications from the LPE in accurately determining $T_{C}$, we continue to use $\mathrm{XeF}_{2}$ released Ir arrays for the thermistors, as these elemental superconductors have not demonstrated that the LPE produces a different $T_{C}=135 \mathrm{mK}$ between a thermistor of $100 \mu \mathrm{m}$ length versus that from a $10 \mu \mathrm{m}$ wide film. However, we may expect a $\pm 10 \mathrm{mK}$ variation in $T_{C}$ due to fabrication uniformity issues, adding a small bit of uncertainty. The arrays for Ir measured here are the same design reported in Ref.[7] and shown in Fig. 3, where 1 $\mathrm{mm}$ long straight beams and $2 \mathrm{~mm}$ long meander beams are compared. In our single-pixel test system, both types of devices can be measured. For our array test system, we had to speed up the devices using a $\mathrm{BOE}$ dip to remove $\mathrm{a} \mathrm{SiO}_{\mathrm{x}}$ layer protecting the devices from $\mathrm{XeF}_{2}$ for improved speed, as is described more fully below. Such devices exhibited a $\mathrm{R}_{\mathrm{N}} \sim 100 \mathrm{~m} \Omega$, almost double that of the devices released using only $\mathrm{XeF}_{2}(55 \mathrm{~m} \Omega)$. Notably, $\mathrm{T}_{\mathrm{C}}$ exceeded or was equal to $160 \mathrm{mK}$, suggesting an increased value due to thinning of the $\mathrm{Ir}$ or another interaction with the BOE.

\section{RESULTS}
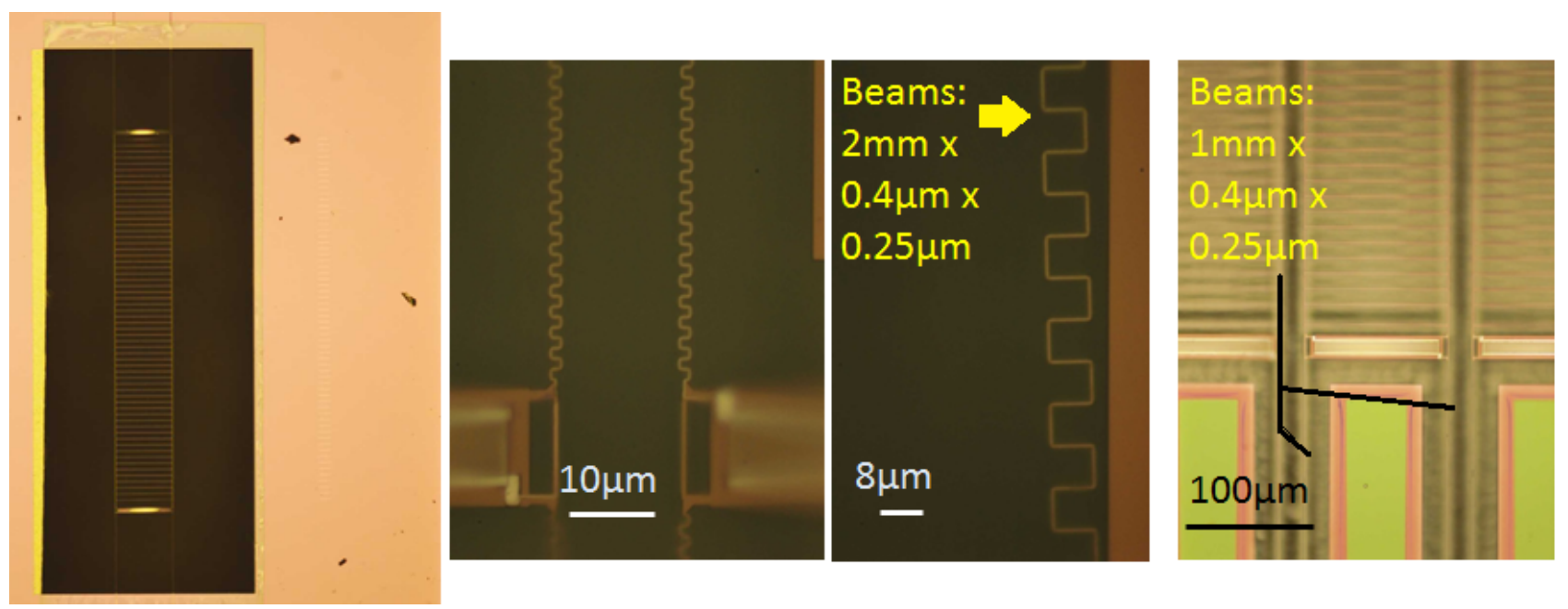

Figure 3: BLISS TESs: (Left) Prototypical image of a BLISS TES plus ladder like absorber. The thermistor sits on either end of the device on the thicker top or bottom rungs of the ladder. (Left middle) Image of a meander beam TES with no absorber. The meander beams are evident as well as the Si-N thermistor platform. (Right middle) A zoomed in image of the meander beams. (Right) Straight beam TESs with varying absorbers were measured in this report. In this image, the BLISS ladder absorber is shown and is suspended by straight beams. 
Previously, we had been unable to measure $\mathrm{NEP}=1.5 \times 10^{-19} \mathrm{~W} / \mathrm{Hz}^{1 / 2}$ in our array test system, as was expected to be observed for the Ir meander TESs. In order to investigate the source of this issue, we measured the amplifier noise in the MUX05 and MUX09 chips in our array test system. We discovered a large 1/f signal with noise equivalent current $\mathrm{NEI}=100 \mathrm{pA} / \mathrm{Hz}^{1 / 2}$ at $0.1 \mathrm{~Hz}$ and $40 \mathrm{pA} / \mathrm{Hz}^{1 / 2}$ to $50 \mathrm{pA} / \mathrm{Hz}^{1 / 2}$ at $1 \mathrm{~Hz}$. Given that we expect the NEI as we enter the transition at high $\mathrm{R}$ to be given approximately by $\sqrt{ }\left(4 \mathrm{k}_{\mathrm{B}} \mathrm{T}_{\mathrm{C}} /\left(\mathrm{R}_{\mathrm{N}} / 2\right)\right) \sim 17 \mathrm{pA} / \mathrm{Hz}^{1 / 2}$, our devices must be much faster than $1 \mathrm{~Hz}$ before we will see this noise above the amplifier/system noise. Otherwise, we will have to drive the devices to much lower values of $\mathrm{R}$, where there is a danger of the device becoming unstable and switching to the superconducting state. ${ }^{11}$
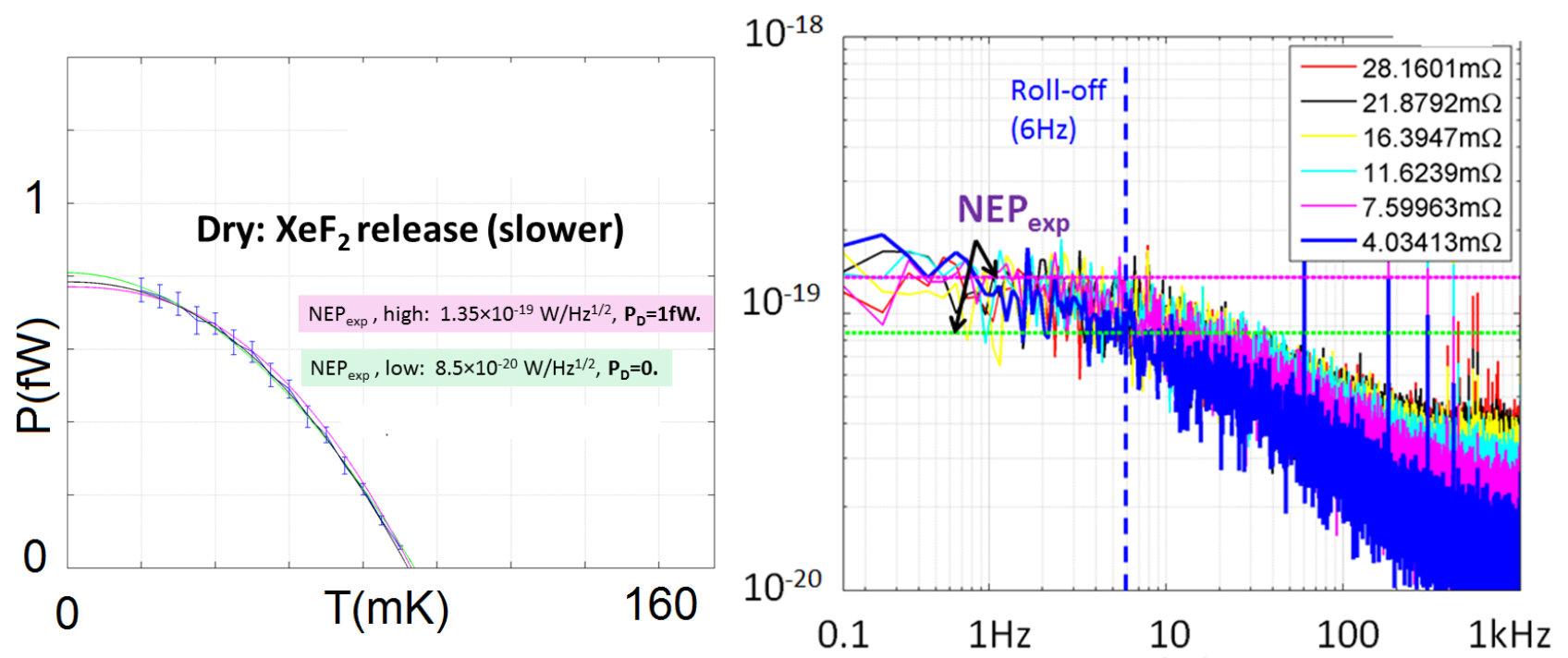

Figure 4: (Left) Measured values of the Joule power $P$ versus temperature in a TES released by $\mathrm{XeF}_{2}$ only. We find $G=(27 \pm 8) f W / K, n=(1.2 \pm 0.2)$, and $T_{C}=125 \mathrm{mK}$ from fitting the data with $E q$. (4) and comparing with the measured NEP. The solid lines illustrate fits to the data using Eq. (4): A fit assuming $G=33 \mathrm{fW} / K, T_{C}=135 \mathrm{mK}$, and $P_{D}=1 \mathrm{fW}$ is shown in magenta. We also include example fits for $P_{D}=0, G=17 \mathrm{fW} / \mathrm{K}$, and $T_{C}=98 \mathrm{mK}$ (green) and $P_{D}=800 \mathrm{aW}, G=27 \mathrm{fW} / \mathrm{K}$, and $T_{C}=125 \mathrm{mK}(\mathrm{blue}$ ), along with the expected NEP for the magenta and green fits- the blue fit expected NEP is $1.35 \times 10^{-19} \mathrm{~W} / \mathrm{Hz}^{1 / 2}$. The estimated value of $P_{D}$ varied between $600 \mathrm{aW}$ to $800 \mathrm{aW}$, for $n=(1.2 \pm 0.2$. (Right) We measured a response time of $\sim 25 \mathrm{~ms}$ in the transition, producing an expected roll-off at $\sim 6 \mathrm{~Hz}$. We measured $\mathrm{NEP}=(1.3 \pm 0.2) \times 10^{-19} \mathrm{~W} / \mathrm{Hz}^{1 / 2}$ from the shown data. The resistances shown in the legend are the values of $R$ within the transition for each NEP curve.

In our single-pixel test system, we measured TES load curves and converted them into power versus resistance curves for a $\mathrm{XeF}_{2}$ released, meandered Ir TES. From the load curves, we were able to obtain the power needed to bias the TESs within their transition $(\mathrm{P})$. The resulting power versus temperature curve, along with error bars due to the uncertainty in $P$ are shown on the left side of Fig. 4. From such a plot and using the uncertainty in $P$, we find $G=(27 \pm 5) f W / K$, and $\mathrm{n}=(1.2 \pm 0.2)$, assuming $\mathrm{T}_{\mathrm{C}}=125 \mathrm{mK}$ from fitting the data with Eq. (4). The solid lines in Fig. 4 illustrate fits to the data using Eq. (4) and different values of $P_{D}$ and $T_{C}$. A fit assuming $G=33 \mathrm{fW} / \mathrm{K}, \mathrm{T}_{\mathrm{C}}=135 \mathrm{mK}$, and $P_{D}=1 \mathrm{fW}$ is shown in magenta. Other example fits shown in Fig. 4 include $P_{D}=0, G=17 \mathrm{fW} / K$, and $T_{C}=98 \mathrm{mK}$ (green) and $P_{D}=800 \mathrm{aW}$, $\mathrm{G}=27 \mathrm{fW} / \mathrm{K}$, and $\mathrm{T}_{\mathrm{C}}=125 \mathrm{mK}$ (blue), along with the expected NEP for the magenta and green fits - the blue fit has an expected phonon NEP $=1.35 \times 10^{-19} \mathrm{~W} / \mathrm{Hz}^{1 / 2}$. All the curves shown could be used to fit the data, illustrating that $\mathrm{T}_{\mathrm{C}}$ needs to be known to accurately in order to determine $P_{D}$. Here, we might expect $T_{C}$ to vary by $\pm 10 \mathrm{mK}$, so our assumption is that the blue curve is the most accurate fit, as the NEP for this fit lies on the measured values while the fit to $135 \mathrm{mK}$ has an expected NEP that is above the measured NEP data. The values of $n$ are consistent with the device being in a crossover regime between the $\mathrm{G} \sim \mathrm{T}^{3}$ for high temperatures and $\mathrm{G} \sim \mathrm{T}^{1 / 2}$ for low temperatures expected for BLISS devices. ${ }^{1}$ The estimated value of $P_{D}$, based on previous performance in the test system, would be between $0-1 \mathrm{fW}$, and assuming $\mathrm{T}_{\mathrm{C}}=125 \mathrm{mK}, \mathrm{P}_{\mathrm{D}}=600 \mathrm{aW}$ to $800 \mathrm{aW}$ for $\mathrm{n}=(1.2 \pm 0.2)$. The NEP measured was computed by multiplying the measured NEI plots multiplying by the simplified expected response $V_{\text {bias }}\left(R-R_{s h}\right) / R$, where $V_{\text {bias }}$ is the voltage bias on the device, $R$ is 
the TES resistance within the transition, and $\mathrm{R}_{\mathrm{sh}}$ is the shunt resistance. We measured a response time of $\sim 25 \mathrm{~ms}$ in the transition, producing an expected roll-off at $\sim 6 \mathrm{~Hz}$. From the plot of NEP versus frequency on the right of Fig. 4 , we report measured $\mathrm{NEP}=(1.3 \pm 0.2) \times 10^{-19} \mathrm{~W} / \mathrm{Hz}^{1 / 2}$.
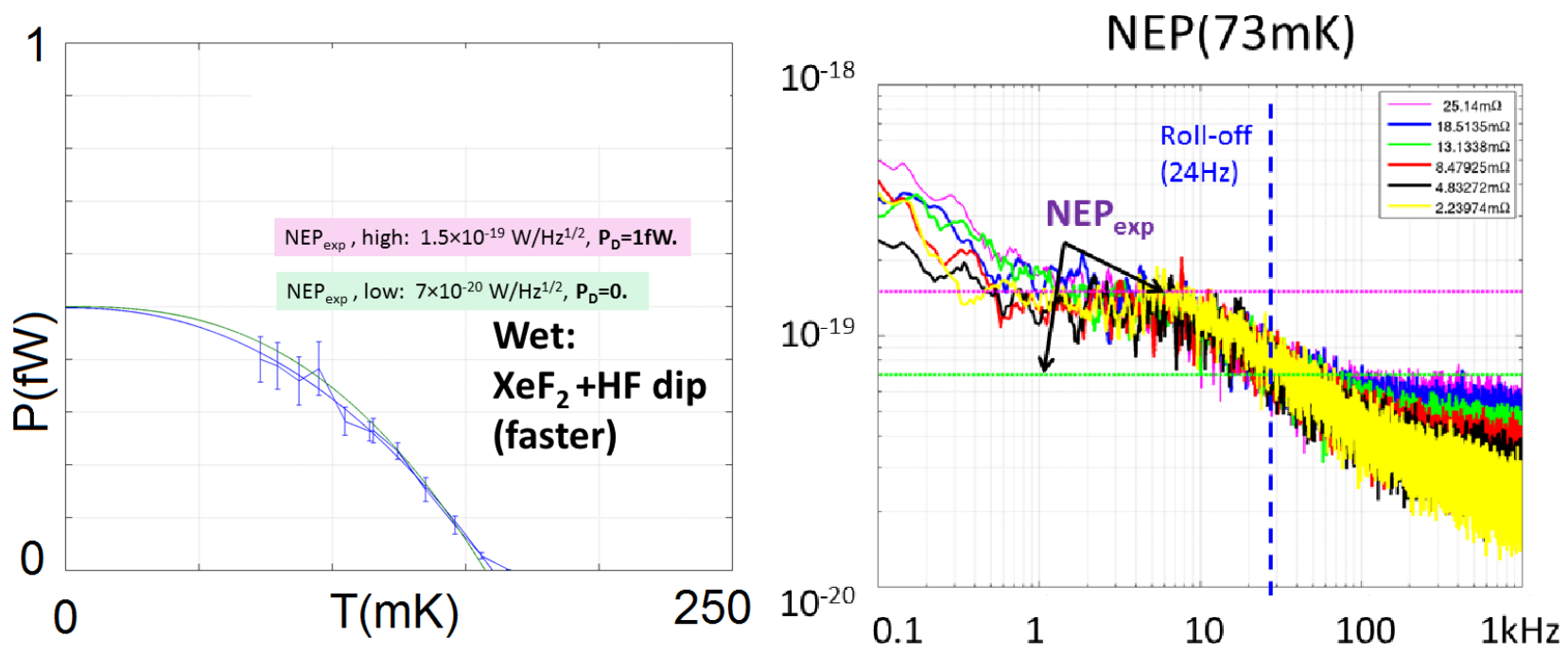

Figure 5: (Left) $P$ vs $T$ curves for a $\mathrm{XeF}_{2}$ released and BOE (HF) dipped, meandered Ir TES. We observed that the normal state resistance of the device was $100 \mathrm{~m} \Omega$ and $T_{C} \geq 160 \mathrm{mK}$, indicating an effect from the BOE. The error bars in $P_{J}$, are shown with example fits with $P_{D}=0, G=7 \mathrm{fW} / K, T_{C}=160 \mathrm{mK}$ and $n=1.2$ (green) and $P_{D}=1 \mathrm{fW}, G=23 \mathrm{fW} / \mathrm{K}, T_{C}=250 \mathrm{mK}$, and $n=1.6(\mathrm{blue})$, along with the expected NEP from Eq. (4). (Right) An excess noise bump near $24 \mathrm{~Hz}$ was observed, implying a factor of 4 increase in speed due to the BOE dip. The measured NEP was $(1.6 \pm 0.3) \times 10^{-19} \mathrm{~W} / \mathrm{Hz}^{1 / 2}$ for this device. The resistances shown in the legend are the values of $R$ within the transition for each NEP curve.

In our array test system, we measured TES load curves and converted them into power versus resistance curves for a $\mathrm{XeF}_{2}$ released and BOE (HF) dipped, meandered Ir TES. We scanned the array and picked the device with the lowest value of $\mathrm{G}$ observed. The resulting power versus temperature curve, along with error bars due to the uncertainty in $\mathrm{P}$ are shown in the left side of Fig. 5. We observed that the normal state resistance of the device was $100 \mathrm{~m} \Omega$, almost double that of the typical $R_{N}$ value of the $\mathrm{XeF}_{2}$ released Ir TESs of $55 \mathrm{~m} \Omega$. We attribute the larger $\mathrm{R}_{\mathrm{N}}$ and increased $\mathrm{T}_{\mathrm{C}}$ to the BOE dip, which most likely thinned the thermistor. The error bars in $\mathrm{P}$, as well as a few example fits with $\mathrm{P}_{\mathrm{D}}=0$, $\mathrm{G}=7 \mathrm{fW} / \mathrm{K}, \mathrm{T}_{\mathrm{C}}=160 \mathrm{mK}$ and $\mathrm{n}=1.2$ (green) and $\mathrm{P}_{\mathrm{D}}=1 \mathrm{fW}, \mathrm{G}=23 \mathrm{fW} / \mathrm{K}, \mathrm{T}_{\mathrm{C}}=250 \mathrm{mK}$, and $\mathrm{n}=1.6$ (blue), along with the expected phonon NEP, are also shown on the left of Fig. 5. Without knowledge of $T_{C}$, we cannot say what the value of $\mathrm{P}_{\mathrm{D}}$ was for this measurement. The NEP measured was computed in the same way as described above. The excess noise bump near $24 \mathrm{~Hz}$ suggests roll-off occurs near this frequency, indirectly showing a factor of 4 increase in speed due to the BOE dip. The measured NEP was $(1.6 \pm 0.3) \times 10^{-19} \mathrm{~W} / \mathrm{Hz}^{1 / 2}$ for this device.

In conclusion, we have measured NEP levels extremely close to the requirement level of BLISS at $10^{-19} \mathrm{~W} / \mathrm{Hz}^{1 / 2}$. The speed of the TES measured here are for TESs with no absorber - we simply used a Si-N platform with enough space to accommodate the thermistor. The absorbers for BLISS resemble ladders (see Ref. [1]). We estimate a roll-off in response at about $5 \mathrm{~Hz}$ to $6 \mathrm{~Hz}$ for the largest band of BLISS based on the BOE dipped device performance. Such an estimate of the roll-off is accomplished by scaling the size of the thermistor platform to that of the entire volume of Si-N expected in the largest band of BLISS including the thermistor platform and the ladder-like absorber. Achieving a lower NEP can be accomplished by producing Ir thermistors with $T_{C}$ close to that of the bulk at $112 \mathrm{mK}$, while maintaining the level of speed observed in the BOE dipped devices. We will investigate this possibility. New $\mathrm{Mo} / \mathrm{Cu}$ devices with lower $\mathrm{T}_{\mathrm{C}}$ and smaller $\mathrm{R}_{\mathrm{N}}$ will be further investigated to achieve the goal NEP and speed necessary for BLISS. Finally, we are working to measure the stray power in our SQUID amplifiers to see if they are the source of the $\sim 1 \mathrm{fW}$ observed here. Determining the source of the stray power will be crucial in order to achieve $\mathrm{P}_{\mathrm{D}}<200 \mathrm{aW}$.

(C) 2012. California Institute of Technology. Government sponsorship acknowledged. 


\section{REFERENCES}

[1] Beyer, A.D., et.al. "Chraracterizing SixNy absorbers and support beams for far-infrared/sub-mm transition-edge sensors," Proc. SPIE 7741, 774121 (2010).

[2] Bradford, C.M. et.al,. "WaFIRS: a waveguide far-IR spectrometer:enabling spectroscopy of high-z galaxies in the far-IR and submillimeter," Proc. SPIE 4850, 1137-1148 (2003).

[3] Houck, J.R. et. al., "The Infrared Spectograph (IRS) on the Spitzer Space Telescope," The Astrophysical Journal Supplement Series 154, 18-24 (2004).

[4] K.D. Irwin, "An application of electrothermal feedback for high resolution cryogenic particle detection," App. Phys. Lett. 66, 1998-2000 (1995).

[5] Stiehl, G.M., et.al. "Time division SQUID multiplexers with reduced sensitivity to external magnetic fields," IEEE Trans. On Appl. Supercon., 21, 298-301 (2011).

[6] Beyer, A.D. et.al. "Characterization of an ultrasensitive transition-edge sensor for space-borne far-IR/sub-mm spectroscopy," IEEE Trans. On Appl. Supercon., 21, 199-202 (2011).

[7] Beyer, A.D., et.al., "Ultra-sensitive transition-edge sensors for the Background-Limited Infrared/Sub-mm Spectrograph (BLISS),” J. Low. Temp. Phys., 167, 182-187 (2012).

[8] Eccosorb ${ }^{\circledR}$, MCS, available from Emerson and Cuming.

[9] Sadleir, J.E., et.al., "Longitudinal proximity effects in superconducting transition-edge sensors," Phys. Rev. Lett., 104, 047003 (2010).

[10] Martinis, J.M., et.al., "Calculation of $T_{C}$ in a normal-superconductor bilayer using the microscopic-based Usadel theory," Nuclear Instruments and Methods in Physics Research Section A, 444, $23-27$ (2000).

[11] Irwin, K.D. and Hilton, G.C., "Transition-edge sensors" in Cryogenic Particle Detection, Topics Appl. Phys. 99, 63152 (2005). 